\title{
Time-domain survey of the Galactic anti-center using Nanshan $1 \mathrm{~m}$ telescope
}

\author{
T.-Z. Yang ${ }^{1,2}$, A. Esamdin ${ }^{1}$, J.-N. Fu ${ }^{3}$, L. Ma ${ }^{1}$, J.-Z. Liu ${ }^{1}$ and \\ F.-F. Song ${ }^{1,2}$ \\ ${ }^{1}$ Xinjiang Astronomical Observatory, CAS, \\ Urumqi, Xinjiang, China \\ email: aliyi@xao.ac.cn \\ ${ }^{2}$ University of Chinese Academy of Sciences, \\ Beijing, China \\ email: yangtaozhi@xao.ac.cn \\ ${ }^{3}$ Department of Astronomy, Beijing Normal University, \\ Beijing, China
}

\begin{abstract}
Following the LAMOST Spectroscopic Survey and Xuyi's Photometric Survey of the Galactic anti-center (GAC), we plan to conduct a time-domain survey of GAC to study the variable sky using Nanshan $1 \mathrm{~m}$ telescope. The survey will be conducted during winter (in Nov., Dec., and Jan.). The first goal of the survey is to cover 270 sq.deg. of sky area in three years. The survey intends to detect some strong transient events of stars and find some short time-scale variable stars of different types. In this paper, we introduce the survey and present the preliminary results already carried out.
\end{abstract}

Keywords. surveys, techniques: photometric, stars: binaries, oscillations.

\section{Introduction}

Time-domain astronomy, which opens the "time axis" of the parameter space, is one of the most promising fields of recent astronomy. Though, the large-sized (about 10 meters) telescope, or the larger (such as VLT and the promising TMT et al.) can explore the deeper and deeper sky in our universe, the small and medium-sized telescopes still have an irreplaceable role, especially in time-domain astronomy. Time-domain survey using the ground based small telescope will strength our understanding of a number of interesting know phenomena, and may lead to the discoveries of unknown ones (Djorgovski et al. 2011).

Nanshan $1 \mathrm{~m}$ telescope is constructed in 2013. Since then, several projects, including study of pulsating stars, eclipsing binaries, stellar population and searching for exoplanets, have been carried out using this telescope. With its modest sensitivity and relatively large field of view, the telescope is a key equipment of Xinjiang Astronomical Observatory (XAO) to conduct time-domain survey.

\section{Nanshan 1m Telescope}

Nanshan $1 \mathrm{~m}$ telescope is located at Nanshan Station of XAO, with coordinates $87.17^{\circ} \mathrm{E}$, $43.30^{\circ} \mathrm{N}$. The medium value of seeing at the site is $1.6^{\prime \prime}$ based on a long time monitoring. The telescope sits on an alt-azimuth mount, and it is primary focus design. The primary mirror of the telescope is $1023 \mathrm{~mm}$ in diameter and prime focal length is $2159 \mathrm{~mm}$. It is equipped with standard Johnson-Cousin-Bessel and Strömgren multicolor filter systems and a liquid nitrogen cooled CCD camera mounted on the primary focus. The CCD 


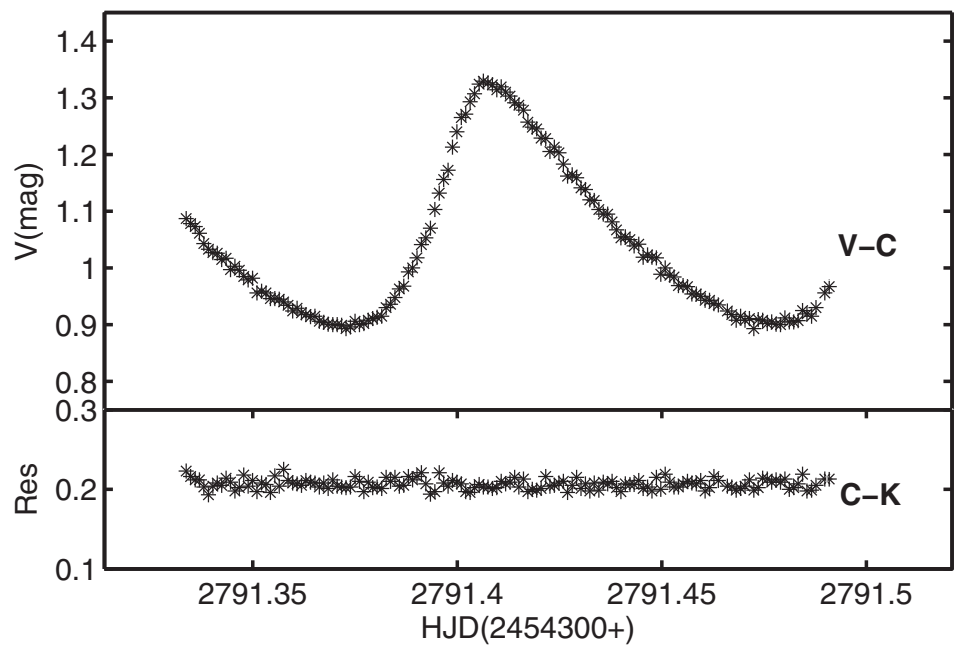

Figure 1. Light curves of $\mathrm{YZ}$ Boo in the $\mathrm{V}$ band observed using Nanshan $1 \mathrm{~m}$ telescope of XAO in Mar 2015. Top panel shows the differential magnitude between reference star and YZ Boo. The standard deviation of the differential magnitude between reference star and check star (bottom panel) yields a general observational accuracy of $0^{m} .006$.

camera (E2V CCD203-82 (blue) chip) has $4096 \times 4096$ pixels, corresponding to a field of view (FOV) of $1.3^{\circ} \times 1.3^{\circ}$ at a focal ratio of 2.159 . The spatial resolution is $1.12^{\prime \prime}$ pixel $^{-1}$, which is matched the seeing of the site.

\section{Observing of Variable stars}

From May 2013, we observed several binaries and variable stars, including YZ Boo (e.g. Boonyarak et al. 2011), XX Cyg (e.g.,Yang et al. 2012), BL Cam (e.g.,Fu et al. 2008), AN Lyn (e.g.,Zhou et al. 2006), EH Lib (e.g.,Wilson et al. 1993). All observations were made through standard Johnson filters. The goal of these observations is to investigate stellar variability and determine its evolution tracks.

A data reduction pipeline was developed based on PyRAF, and real-time data reduction has been made to all the observations. Fig. 1 shows the light curves of YZ Boo in the Johnson $\mathrm{V}$ band observed with Nanshan 1m telescope in 2015. A general observational accuracy is $0^{m} .006$. For binary star LO COM, the final light curves have been plotted in phase and are shown in Fig. 2. The light curves are typical EW type, and present symmetry feature in three bands, indicating absence of the $\mathrm{O}^{\prime}$ Connell effect. Detailed study and discussion on these variables will be in forthcoming papers.

\section{Time-domain Survey of the Galactic Anti-center (GAC) using Nanshan 1m Telescope}

Following the LAMOST (Cui et al. 2012) Spectroscopic Survey of the Galactic Anticenter (LSS-GAC; Liu et al. 2013) and Xuyi Schmidt Telescope Photometric Survey of the Galactic Anti-center (XSTPS-GAC; Zhang et al. 2014), we plan to conduct a timedomain survey of GAC (TDS-GAC) using Nanshan 1m telescope. The survey intend to detect some strong transient events of stars and find some short time-scale variable stars of different types. 


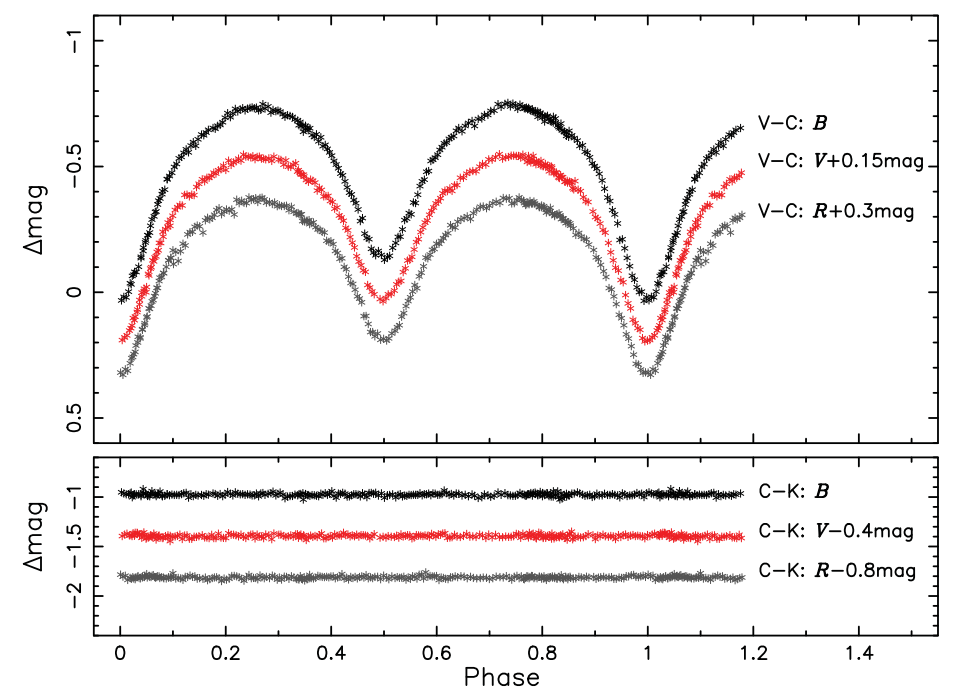

Figure 2. Light curves of LO COM in the B, V \& R bands observed using Nanshan $1 \mathrm{~m}$ telescope of XAO in Mar 2015. The light curves are typical EW type, and present symmetry feature in three bands, indicating absence of the O' Connell effect.

The LSS-GAC surveys a significant volume of the Galactic thin/thick disks and halo in a contiguous sky area of $\sim 3,400$ sq.deg., centered on the Galactic anti-center $\left(|\mathrm{b}| \leqslant 30^{\circ}\right.$, $150^{\circ} \leqslant l \leqslant 120^{\circ}$ ). LSS-GAC aims to study stellar populations, disk structures, chemical composition and kinematics of the thin/thick disks, and so on, to ultimately advance our understanding of the assemblage of galaxies and the origin of their regularity and diversity (Liu et al. 2013).

The XSTPS-GAC is an important photometric component of the Digital Sky Survey of the Galactic Anti-center, which is a photometric and spectroscopic sky survey, in combination with LAMOST (Zhao et al. 2012). The survey was initiated in the fall of 2009 and completed in the spring of 2011. XSTPS-GAC collected more than 20000 CCD images in the three Sloan Digital Sky Survey $g$-, $r$ - and $i$ - bands, covering a continuous area of sky over 6000 sq.deg. toward GAC and an extended area of about 900 sq.deg. toward M31/M33 (Zhang et al. 2014).

As an important supplement for above surveys, we plan to start a systematic TDSGAC using Nanshan $1 \mathrm{~m}$ telescope. The survey will be carried out in grey and dark nights through Nov., Dec. and Jan.. Crossing the middle of LSS-GAC survey area, we design 3 observation stripes, each of constant declination. One strip will be observed in one winter. In a given night, a field of about 1 sq.deg. in stripe will be continually observed; in the following night, the field center will shift $1^{\circ}$ in RA, yielding an overlap of $\sim 18$ arcmin between two adjacent fields. The stepping in DEC between the stripes set at $1.1^{\circ}$, yielding an overlap of $\sim 12$ arcmin between two adjacent stripes. The exposure time of each field is $60 \mathrm{sec}$. With four-channel readout time of $25 \mathrm{sec}$, the sampling time of one frame is about $85 \mathrm{sec}$. With $8 \sim 12$ hours continuous observation of one field per night during the winter at the site, some interesting short time-scale (minutes $\sim$ hours) transient variations of stellar may be detected. 


\section{Summary}

TDS-GAC is a project of three years conducted with Nanshan $1 \mathrm{~m}$ telescope. The project may provide us with an opportunity to deepen our understanding of the behavior of know variable stars and discover some new and interesting short time-scale variation phenomena of celestial objects. Together with spectroscopic data of LSS-GAC and photometric data of XSTPS-GAC, the TDS-GAC database of same area will yield an unique data set to make an in-depth study of stellar astrophysics in the time-domain.

\section{Acknowledgement}

This work is funded by the National Natural Science Foundation of China (NFSC) under No. 11273051.

\section{References}

Boonyarak. C., Jing, S. Y., \& Khokhuntod, P., Fu. J. N. 2011, Ap\&SSS, 125,131

Cui, X.-Q., Zhao, Y.-H., \& Chu, Y.-Q., et al. 2012, RAA, 12, 1197

Djorgovski, S. G., et al. 2011, IAU Symposium, 285, 2011

Fu, J.-N., Zhang, C., \& Marak, M., et al. 2008, ChjAA, 8, 237

Liu, X. -W., et al. (2013), IAU Symposium, 298, 2013

Ward, R., Delaney, P., \& Sadavoy, S., et al. 2008, JRASC, 102,134

Wils, P., Kleidis, S., \& Hambsch, F. J. 2009, IBVS, 5878,2009

Yang, X. H, Fu, J. N., \& Zha, Q 2012, AJ, 144, 92

Zhao, G., Zhao, Y.-H., Chu, Y.-Q., Jing, Y.-P., \& Deng, L.-C. 2012, RAA, 12, 723

Zhang, H.-H., Liu, X.-W., Yuan, H.-B., et al. 2014, RAA, 14, 456

Zhou, A. Y. 2006, Ap\&SS, 301: 11-20 